Recepción: 25 / 03 / 2018

Aceptación: 27 / 04 / 2018

Ciencias económicas y empresariales

Publicación: 01 / 08 / 2018

Artículo de investigación

\title{
El marketing relacional y la fidelización del cliente
}

\author{
Relationship marketing and customer loyalty
}

Marketing de relacionamento e fidelização de clientes

Ángel B. Burbano-Pérez I bolobur@hotmail.com

Ernesto B. Velástegui-Carrasco II vireymo@hotmail.es

Jenny M. Villamarin-Padilla ${ }^{\text {III }}$ nyvillamarin@yahoo.es

Carlos E. Novillo-Yaguarshungo IV carlitosnovillo@ hotmail.com

Correspondencia: bolobur@ hotmail.com

\footnotetext{
${ }^{I}$ Magister en Pequeñas y Medianas Empresas Mención Finanzas, Licenciado en Comunicación Social, Ingeniero Industrial, Tecnólogo Mecánico, Docente de la Escuela Superior Politécnica de Chimborazo, Riobamba, Ecuador.

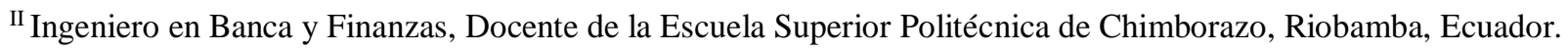
III Magister en Matemática Básica, Doctora en Matemática, Docente de la Escuela Superior Politécnica de Chimborazo, Riobamba, Ecuador.

${ }^{\text {IV }}$ Magister en Gestión Industrial y Sistemas Productivos, Ingeniero de Mantenimiento, Tecnólogo en Mantenimiento Industrial, Docente de la Escuela Superior Politécnica de Chimborazo, Riobamba, Ecuador.
} 


\section{Resumen}

La concepción del marketing ha evolucionado con la flexibilidad que requieren hoy en día las ciencias administrativas empresariales, a tal punto que a lo largo del tiempo ha ido tomando aspectos relevantes y necesarios para la innovación de esta área. Esto constituye el origen del marketing relacional, al incluir en el concepto general la relación que debe existir entre la empresa y el cliente y de la cual nace la fidelización que es su propósito principal. La presente investigación se realizó mediante una revisión documental bibliográfica. El marketing relacional, entendido hoy en día, se centra en formar y mantener una relación entre el cliente y la empresa, basada en la personalización de la atención, la recolección de datos y el apoyo fundamental en el cliente, con el propósito de generar confianza, satisfacción, una relación duradera, información importante para la toma de decisiones, recomendación y ampliación de la cartera de cliente, entre otros. Se concluye, que el objetivo fundamental del marketing relacional es la fidelización del cliente por cuanto es el garante de una relación firme, satisfactoria y duradera con miras a incrementar las ventas y el éxito, en general, de la empresa.

Palabras claves: marketing; relacional; fidelización; objetivo; cliente.

\section{Abstract}

The conception of marketing has evolved with the flexibility required today by the administrative business sciences, to the point that over time it has taken relevant and necessary aspects for innovation in this area. This is the origin of relational marketing, by including in the general concept the relationship that must exist between the company and the client and from which loyalty is born, which is its main purpose. The present investigation was carried out through a bibliographic documentary review. Relationship marketing, understood today, focuses on forming and maintaining a relationship between the client and the company, based on the personalization of attention, data collection and fundamental support in the client, with the purpose of generating trust, satisfaction, a lasting relationship, important information for decision making, recommendation and expansion of the client portfolio, among others. It is concluded that the fundamental objective of relationship marketing is customer loyalty as it is the guarantor of a firm, satisfactory and lasting relationship with a view to increasing sales and the success, in general, of the company. 
Keywords: marketing; relational; loyalty objective; client.

\section{Resumo}

A concepção de marketing evoluiu com a flexibilidade exigida hoje pelas ciências administrativas, a ponto de, com o passar do tempo, ter assumido aspectos relevantes e necessários para a inovação nessa área. Esta é a origem do marketing de relacionamento, através da inclusão no conceito geral da relação que deve existir entre a empresa e cliente é nascido e lealdade que é o seu principal objectivos. A presente investigação foi realizada por meio de uma revisão documental bibliográfica. Marketing de relacionamento, entendida hoje, se concentra na construção e manutenção de um relacionamento entre o cliente e empresa, com base em personalização do atendimento, coleta de dados e apoio crítico no cliente, a fim de construir a confiança, satisfação, relacionamento duradouro, informações importantes para tomada de decisão, recomendação e ampliação da carteira de clientes, entre outros. Conclui-se que o objetivo principal do marketing de relacionamento é a fidelidade do cliente, porque ele é o fiador de uma relação forte, bem sucedida e duradoura com vista a aumentar as vendas e sucesso em geral da empresa.

Palavras chave: Marketing; Relacional Lealdade; Objetivo Cliente.

\section{Introducción}

A través del tiempo el marketing como muchos conceptos han sufrido cambios y adaptaciones, logrando mantener su definición acorde con la aplicación que requiere para cada etapa.

Cobo \& González (2007), citan a Cobo \& Aguado (2006), para hacer mención a la evolución del concepto de marketing a lo largo del tiempo, el cual se ha ido adaptando a los nuevos retos de cada época y a las exigencias nacientes de las sociedades. En tal sentido, la American Marketing Associatión (AMA) sirve de foro a los conceptos emergentes, constantemente actualiza los conceptos de esta área y no es sino hasta 2004 donde integra en su definición la filosofía del marketing relacional. (p. 545)

Este fue el inicio del marketing relacional, a partir de la inclusión del estudio y la relación existente entre la empresa y el cliente y la importancia que repercute para la organización. 
Gómez y Uribe, (2016) citan a Shaw \& Jones (2005), para mencionar que cerca de la mitad del siglo pasado, se dio un cambio de paradigma que colapsó el enfoque tradicional del concepto de marketing y que empiezan a incluirse escuelas que tratan la gestión, sistemas de marketing, comportamiento del consumidor, neuromarketing, intercambio y ventas. Más tarde, hacia 1975, tres de las escuelas, cambiaron su direccionamiento y se empieza a analizar la conducta social humana, por lo que, en pleno siglo XXI, se empieza a entender qué es lo que busca el cliente.

En este sentido, tenemos que el marketing actual se preocupa por el cliente de diferentes formas, desde lo que quiere, lo que busca, lo que necesita, la forma en que le gustaría adquirirlo, sus expectativas, la medición de su satisfacción, entre otros aspectos, que representarán la base de datos de donde partirán la elaboración de las estrategias necesarias para ofrecer un producto o servicio que cumpla con las expectativas y que genere no sólo la satisfacción sino la fidelidad del cliente.

Por otra parte, Cobo Quesada \& González Ruíz (2007), afirman acerca del marketing relacional "presenta un enfoque que se inicia con las investigaciones del marketing en el sector de servicios y en el sector industrial y que en los últimos años ha tenido un gran impulso en el sector del gran consumo gracias a las posibilidades que las tecnologías de la información y la comunicación han aportado”. (p. 545)

La importancia del marketing relacional radica en las relaciones establecidas con los clientes, las cuales deben ser satisfactorias, las emociones y la confianza juegan un papel fundamental en la creación de fidelidad en el cliente a lo largo del tiempo. Un cliente satisfecho y fiel es el origen de múltiples beneficios para la empresa ya que ellos, por medio de la información que generan construyen la base de datos sobre la cual la empresa planifica las estrategias a seguir para garantizar su crecimiento y en líneas generales el alcance de sus metas.

La presente investigación plasma la evolución del concepto de marketing relacional y analiza su flexibilidad e importancia a lo largo del tiempo, asimismo, hace énfasis en la importancia de su aplicación. Por otra parte, estudia la fidelización como objetivo principal del marketing relacional, así como la puntualización de aspectos que las empresas deben tomar en cuenta en la actualidad para lograr un cliente fiel y satisfecho. 


\section{Metodología}

La investigación se desarrolló mediante la recopilación y revisión de material documental bibliográfico, a través del cual se logró plasmar aspectos relacionados con la evolución del concepto de marketing relacional y la fidelización como objetivo fundamental de esta rama del marketing. Al respecto, Tancara (1993), define la investigación documental como "una serie de métodos y técnicas de búsqueda, procesamiento y almacenamiento de la información contenida en los documentos, en primera instancia, y la presentación sistemática, coherente y suficientemente argumentada de nueva información en un documento científico, en segunda instancia" (p. 94)

\section{Resultados}

\section{Marketing Relacional (Conceptos)}

Una de las primeras definiciones de marketing relacional la plasma Córdoba López, (2009) quien cita a Berry (1983), el cual establece que "el marketing relacional consiste en atraer, mantener e (en las organizaciones multiservicios) intensificar las relaciones con el cliente". (p. 9)

Por su parte, Christopher, Payne, \& Ballantyne (1994), abordan la definición de marketing relacional desde la vinculación de los conceptos de marketing, servicio al cliente y calidad. Manifiestan además que "el marketing se preocupa por las relaciones de intercambio que se producen entre la organización, sus clientes, la calidad y el servicio a la clientela son los eslabones claves en esta relación". Asimismo, indican que estos elementos deben ser tratados entre sí y que esa vinculación debe ser explotada eficaz y efectivamente para alcanzar la total satisfacción de los clientes y el mantenimiento de las relaciones a largo plazo. (p. 4,5)

Vemos como el concepto de marketing relacional ha evolucionado desde el simple estudio de la relación de la empresa con el cliente hasta el estudio del marketing como tal, el servicio al cliente y la calidad en los productos, con el propósito fundamental de satisfacer al cliente y mantener estas relaciones a lo largo del tiempo.

Un concepto más reciente lo definen como "el análisis e implementación permanente de estrategias que lleven a la identificación de los tipos de consumidores con los que cuenta la 
organización y les de atención personalizada, con el fin de lograr relaciones a largo plazo con mejores índices de satisfacción y fidelidad”. (Gómez \& Uribe, 2016)

En este orden de ideas, es imperioso resaltar el protagonismo del cliente dentro de los procesos de la organización y el avocamiento de la misma hacia una atención personalizada con la finalidad de fidelizarlo.

\section{Objetivos}

Córdoba López, (2009) cita a Kotler (2006) el cual argumenta que "el marketing de relaciones tiene por objetivo establecer relaciones mutuamente satisfactorias y de largo plazo, con los participantes clave (consumidores, proveedores, distribuidores y otros socios del marketing) con el fin de conservar e incrementar el negocio". (p. 10)

Ahora bien, de lo anterior se puede extraer dos aspectos fundamentales a saber: por un lado, establecer relaciones satisfactorias y por el otro que estas relaciones perduren en el tiempo. Es importante para las empresas crear una relación con el cliente, ganar su confianza y mantenerla por largos periodos para garantizar el éxito y crecimiento de la empresa.

Para Agüero Cobo, (2014) los objetivos del marketing relacional son: el aumento de las ventas, fidelidad de los clientes, la introducción de nuevos productos, la mejora de la imagen de la empresa, nuevas técnicas de venta, captación de nuevos clientes. (p. 10,11)

Abarcar todos estos aspectos es fundamental en la actualidad para cumplir las metas empresariales, no sólo es buscar la fidelización, sino las mejoras en todos aquellos procesos que llevan a la organización a obtener la satisfacción y mantengan la relación con el cliente a lo largo del tiempo.

"La fidelidad es un objetivo básico del Marketing Relacional y la lograremos cuando vayamos más allá de la satisfacción del cliente. Debemos deleitar al cliente, superar con creces sus expectativas y anticiparnos a sus necesidades”. (Abad, 2018)

\section{Importancia}

La importancia del marketing relacional se centra en establecer una relación simbiótica con el cliente, donde el cliente se beneficia de la empresa y viceversa, y que esta relación se mantenga el 
mayor tiempo posible. Lograr que los clientes existentes sean fieles a la marca e incrementar el número de cliente trabajando en el fortalecimiento de nuevas relaciones, esto lo convierte en un ciclo cerrado, donde no se puede iniciar un proceso (captación de un cliente) sin pensar en su final (fortalecimiento de la relación), de esta forma mantener el mayor número de clientes fieles a la marca.

"Existen quienes afirman que es mejor un cliente que compra 20 veces al año que 10 que compran una vez durante el mismo periodo, pero esto implica depender de un comprador lo cual puede resultar contraproducente para las organizaciones al correr el riesgo de perderle cuando decida ir a la competencia ocasionando problemas de estabilidad económica. Por el contrario, tener demasiados que solo compren una sola vez es un síntoma de desatenciones en el servicio que pueden repercutir en la pérdida de los mismos. En tal sentido, lo mejor es contar con 20 asiduos que compren 20 veces al año y para ello existe el marketing relacional”. (Fernández Domínguez, 2018)

\section{Beneficios}

Para de Guzmán Miranda (2014), quien cita a Reichheld y Sasser (1990), uno de los principales beneficios que se pueden obtener de la aplicación de estrategias de marketing relacional "es la reducción de la tasa de pérdida de clientes. Otro beneficio, se refiere a que un cliente genera más beneficios para la empresa, a medida que la relación entre ambos se hace más duradera. Por último, es que los clientes satisfechos se convierten habitualmente en recomendadores del producto o del servicio. (p. 38)

La fidelización de un cliente abre la brecha para la captación de otros, un cliente satisfecho recomienda la marca, asimismo es más rentable mantener a un cliente que invertir en la captación de clientes nuevos, representa una ventaja invertir en las relaciones a largo plazo ya que lo demás vendrá por añadidura.

\section{Fidelización de los clientes}

Peña, Ramírez \& Osorio (2014), sobre fidelización de clientes hacen una importante diferenciación con el concepto de retención: "fidelidad es un constructo multidimensional más amplio y complejo que el de retención, este último es un indicador del primero, relacionado con 
sentimientos de satisfacción del cliente con el producto o servicio, e impacta positivamente en el comportamiento del cliente". (p. 90)

Guadamarra \& Rosales (2015) de acuerdo con Evans (2002), indican que "para centrarse en el cliente se debe transitar hacia un marketing personalizado; saber qué, cuándo y cómo lo necesitan, y qué precios están dispuestos a pagar los clientes, darles mayores expectativas y hacerlos sentir en el centro de la organización”. (p. 311)

Las nuevas estrategias de marketing relacional apuntan a la personalización del cliente, cada vez son más las muestras de afecto y atención de las empresas a cada particular, ya sea por medio de un correo electrónico o una llamada, donde la premisa es dirigirse al cliente por su nombre y enviar contenido adaptado a su gusto y necesidades. Todo este trabajo requiere de una base de datos cuyo principal instrumento de apoyo son las nuevas tecnologías. Este trato personalizado, hace sentir especial al cliente, convirtiéndolo en parte importante de la organización al ser incorporados a los procesos y productos sus gustos y recomendaciones.

Por otra parte, Guadamarra \& Rosales, citando a Pitta (1998), indican que el marketing personalizado crea lealtad y barreras a la competencia, ello es importante ya que esta estrategia empresarial consiste en la búsqueda, identificación y mantenimiento de los clientes de mayor valor. (p. 312)

Indiscutiblemente el marketing relacional fundado en la satisfacción del cliente y la innovación en la oferta es una estrategia fundamental ante la creciente y desmedida competencia del entorno actual.

Cobo Quesada \& González Ruiz (2007), citan a Vázquez y Trespalacios (1998), para mencionar acerca de la fidelización de clientes que "es uno de los pilares del marketing relacional, posiblemente el más llamativo debido a todas las posibilidades que las nuevas tecnologías permiten para gestionar las relaciones con los clientes”. (p. 554).

Para Alcaide, (2016) alcanzar la fidelización de los clientes requiere las siguientes consideraciones:

Reconocer el creciente poder del cliente, el cliente a través de internet tiene el poder de destruir una marca, es más fácil hablar de lo negativo que de lo positivo, a esta premisa se une la facilidad 
en la difusión de la información por medio del internet. En virtud de ello, es importante reconocer el poder que tiene el cliente sobre el posicionamiento de una marca.

Desarrollar una oferta al mercado objetivo: para lograr lealtad en estos tiempos es necesario personalizar la atención al cliente, a pesar de haber sido abordado con anterioridad este tema, se puede puntualizar que la personalización es una de las estrategias claves del marketing relacional que cada vez toma más relevancia por cuanto crea lazos de afecto y emociones entre la empresa y el cliente.

Diseñar las estrategias del marketing desde la perspectiva del cliente: el cliente necesita ser sorprendido, necesita que sus expectativas sean colmadas, no sólo alcanzadas sino superadas, esta es la garantía del éxito del marketing relacional, quedar bien para generar confianza.

Centrarse en aportar soluciones y resultados, no productos: los productos pueden ser copiados, las emociones no, el foco básico en este aspecto estará en llenar espacios y carencias emocionales a través de soluciones prácticas, no sólo ofrecer el producto sino cómo se usa, cómo se cuida, cual es la opinión del mismo y crear lazos emocionales tomando en cuenta lo que el cliente tiene como aporte de mejoras futuras.

Apoyarse en el cliente para colaborar en la creación de valor: consiste en permitir que el cliente participe activamente de la creación y modificación de procesos, productos y servicios, tomando como medio de comunicación los sociales.

Usar nuevas vías para hacer llegar el mensaje al cliente: es imperioso crear estrategias multicanal, mediante una comunicación multidireccional.

Desarrollar métricas y rigurosas mediciones de ROI: se hace necesario medir para demostrar cuantitativa y cualitativamente la actividad en marketing y fidelización.

Apostar por un marketing científico: por medio del uso de la estadística y la matemática, la psicología clínica y la métrica sociológica, todo aquello que permita demostrar teorías y cuantificar el trabajo.

Desarrollar los activos de largo recorrido en la compañía: la política de fidelización es una aventura de largo plazo, es una relación que tarda años en consolidarse y que se puede demostrar a través de la satisfacción, del compromiso mutuo, de boca a boca, entre otros. 
Implantar en la empresa una visión holística: el marketing debe ser experiencial, emocional, ya no serán solo las 4P (plaza, producto, precio y promoción), hay que sumar procesos cómodos y fidelizadores, crear experiencias dignas de contar, para ello es necesario un personal especializado que será pieza fundamental del marketing de la nueva era.

\section{Conclusión}

Se concluye, por una parte, que el marketing relacional es una ramificación del marketing que ha ido evolucionando en su concepto a lo largo del tiempo y resulta de una definición flexible que incorpora las relaciones entre la empresa y el cliente como estrategia de mantenerse activo en la ruda competencia de la actualidad.

Por otra parte, tenemos que la fidelización es uno de los objetivos fundamentales del marketing relacional, el cual proporciona por medio de una relación de confianza, emocional y satisfactoria, innumerables beneficios para la empresa y que el éxito y crecimiento de la organización está influenciado por la permanencia en el tiempo de esa fidelización en el cliente.

Por último, tenemos que para aplicar estrategias de marketing relacional que permitan fidelizar el cliente y mantenerlo en el tiempo requiere, en términos prácticos, de la consideración de aspectos que devienen de los cambios constantes del entorno empresarial, las nuevas tecnologías representan el pilar fundamental en la planificación y ejecución del marketing relacional, darle el valor que merece el cliente y no subestimar su poder son factores claves para lograr el éxito en cada estrategia, así como la personalización en el trato al cliente es determinante para la fidelización.

\section{Referencias Bibliográficas}

Abad, R. (2018). Raulabad. Recuperado el 10 de agosto de 2018, de https://www.raulabad.com/articulos/marketing-relacional-crm-y-fidelizacion

Aguero Cobo, L. (2014). Universidad de Cantabria. Recuperado el 10 de agosto de 2018, de https://repositorio.unican.es/xmlui/bitstream/handle/10902/4474/\%5B2\%5D\%20Ag\%C3\%BCero $\% 20$ Cobo\%20L.pdf

Alcaide, J. C. (2016). Fidelización de clientes (2da ed.). Madrid: ESIC. Recuperado el 10 de Agosto de 2018, de 
https://books.google.co.ve/books?hl=es\&lr=\&id=87K_CQAAQBAJ\&oi=fnd\&pg=PA11\&dq=ma rketing+relacional+\%22fidelizaci\%C3\%B3n+de+los+clientes\%22\&ots=RlTeRzE0zB\&sig=YYO rMZ1hodkxGZwa8CTiRK0HYVs\#v=onepage \&q=marketing\%20relacional\%20\%22fidelizaci\% C3\%B3n\%20de $\% 2010$ \% $\% 2$

Christopher, M., Payne, A., \& Ballantyne, D. (1994). Marketing relacional: integrando la calidad, el servicio al cliente y el marketing. Diaz de Santos. Recuperado el 10 de agosto de 2018, de https://books.google.co.ve/books?hl=es\&lr=\&id=YMfCGu3B-

$\mathrm{yMC} \& \mathrm{oi}=\mathrm{fnd} \& \mathrm{pg}=\mathrm{PA} 1 \& \mathrm{dq}=$ marketing + relacional\&ots=e5KXCMQWsX\&sig=B3vNQWQQGn jwe7zOunb3znP0I4U\#v=onepage \&q=marketing\%20relacional $\& \mathrm{f}=$ false

Cobo Quesada, F. B., \& González Ruiz, L. (2007). Las implicaciones estratégicas del marketing relacional. Anuario Jurídico y Económico Escurialense (40), 543-568. Recuperado el 10 de Agosto de 2018, de https://dialnet.unirioja.es/servlet/articulo?codigo=2267957

Córdoba López, J. F. (2009). Del marketing transaccional al marketing relacional. Entramado, 5(1). Recuperado el 10 de agosto de 2018, de https://dialnet.unirioja.es/servlet/articulo?codigo=3993098

De Guzmán Miranda, J. C. (2014). Estrategia de marketing relacional para lograr la fidelización de los clientes. Apuntes Universitarios, 4(2), 25-42. Recuperado el 10 de agosto de 2018, de http://www.redalyc.org/pdf/4676/467646129002.pdf

Fernández Domínguez, S. (2018). Universidad de Guadalajara. Recuperado el 10 de agosto de 2018, de http://iditpyme.cucea.udg.mx/?q=noticia/la-importancia-del-marketing-relacional

Gómez, L., \& Uribe, J. (12 de mayo de 2016). Marketing Relacional: La evolución del concepto. Espacios, 36(25), 16. Recuperado el 10 de agosto de 2018, de http://www.revistaespacios.com/a16v37n25/16372511.html

Guadarrama T., E., \& Rosales E., E. (2015). MARKETING RELACIONAL: VALOR, SATISFACCIÓN, LEALTAD Y RETENCIÓN DELCLIENTE. ANÁLISIS Y REFLEXIÓN TEÓRICA. Ciencia y Sociedad, 40(2), 307-340. Recuperado el 10 de agosto de 2018, de http://www.redalyc.org/pdf/870/87041161004.pdf 
Peña E., S., Ramírez R., G. S., \& Osorio G., J. (2014). Evaluación de una estrategia de fidelización de clientes. Ingenierías Universidad de Medellín, 14(26), 87-104. Recuperado el 10 de agosto de 2018, de http://www.scielo.org.co/pdf/rium/v14n26/v14n26a07.pdf

Tancara, C. (1993). La Investigación Documental. Temas Sociales (17), 91-106. Obtenido de http://www.revistasbolivianas.org.bo/pdf/rts/n17/n17a08.pdf. 